Research Square

\title{
A search for a stochastic archetype of quantum probability
}

Tim C Jenkins ( $\nabla$ tjenkins@bigpond.net.au )

Australian Actuaries Institute https://orcid.org/0000-0001-8419-1710

\section{Research Article}

Keywords: quantum probability, interference, hidden variables, archetype, nonlocality

Posted Date: December 16th, 2021

DOI: https://doi.org/10.21203/rs.3.rs-954460/v8

License: (a) (1) This work is licensed under a Creative Commons Attribution 4.0 International License. Read Full License

Version of Record: A version of this preprint was published at Advances in Theoretical \&amp; Computational Physics on April 1st, 2022. See the published version at https://doi.org/10.33140/atcp.04.04.08. 


\title{
A search for a stochastic archetype of quantum probability
}

\author{
Tim C Jenkins \\ Fellow \& Life Member \\ Australian Actuaries Institute \\ 2/50 Carrington Street, Sydney NSW 2000 \\ tjenkins@bigpond.net.au \\ ORCID 0000-0001-8419-1710
}

The author of this article does not have any conflicts of interest in connection with it.

Keywords: quantum probability, interference, hidden variables, archetype, nonlocality

\begin{abstract}
Superposed wavefunctions in quantum mechanics lead to a squared amplitude that introduces interference into a probability density, which has long been a puzzle because interference between probability densities exists nowhere else in probability theory. In recent years, Man'ko and coauthors have successfully reconciled quantum and classic probability using a symplectic tomographic model. Nevertheless, there remains an unexplained coincidence in quantum mechanics, namely, that mathematically, the interference term in the squared amplitude of superposed wavefunctions gives the squared amplitude the form of a variance of a sum of correlated random variables, and we examine whether there could be an archetypical variable behind quantum probability that provides a mathematical foundation that observes both quantum and classic probability directly. The properties that would need to be satisfied for this to be the case are identified, and a generic hidden variable that satisfies them is found that would be present everywhere, transforming into a process-specific variable wherever a quantum process is active. Uncovering this variable confirms the possibility that it could be the stochastic archetype of quantum probability.
\end{abstract}

\section{Introduction}

Using the Schrödinger picture and the causal interpretation as recounted by Peter Holland in his quantum theory of motion, in particular [1],[2],[10],[11], and confining ourselves to a single spatial dimension to keep a focus on concepts, the probability distribution of the location of a moving particle in the spatial dimension $x$ at time $t$ is derived from its wavefunction $\psi(x, t)$, which is defined and continuous everywhere. The squared amplitude of the wavefunction is $|\psi(x, t)|^{2}=\psi(x, t) \psi^{*}(x, t)$, where $\psi^{*}$ is the complex conjugate of $\psi$, and the probability density $f(x, t)$ associated with the location of the particle being between $x$ and $x+d x$ at time $t$ is given by

$$
f(x, t)=\frac{\psi(x, t) \psi^{*}(x, t)}{C(t)}
$$


where $C(\mathrm{t})$ is a normalization constant $\int_{-\infty}^{\infty} \psi \psi^{*} d x{ }^{1}$, which varies with $t$. Provided that $\int_{-\infty}^{\infty} \psi \psi^{*} d x$ is finite, $C(t)$ rescales the squared amplitude to a probability density by satisfying $\int_{-\infty}^{\infty} f(x, t) d x=1$ and reflects the fact that the particle exists somewhere at time $t^{2}$.

The one-dimensional Schrödinger equation is:

$$
i \hbar \frac{\partial \psi}{\partial t}=\left(-\frac{\hbar^{2}}{2 m} \frac{\partial^{2} \psi}{\partial x^{2}}+V\right) \psi
$$

where $m$ is inertial particle mass, $V=V(x, t)$ is the potential energy due to an external classic potential field, $\hbar=\frac{h}{2 \pi}$, and $h$ is Planck's constant [1]. Consider two waves associated with the same physical system and its particle, with two distinct solutions of this equation which expressed in polar form are:

$$
\psi_{1}=R_{1} e^{\frac{i S_{1}}{\hbar}}, \text { and } \psi_{2}=R_{2} e^{\frac{i S_{2}}{\hbar}}
$$

where $R_{1}, R_{2}$ are the respective wave amplitudes, by definition nonnegative real functions of space and time, $\psi_{1} \psi_{1}^{*}=R_{1}^{2}, \psi_{2} \psi_{2}^{*}=R_{2}^{2}$ are the respective squared amplitudes, and $S_{1}, S_{2}$ are the respective wave phases, also real functions of space and time.

The superposition $\psi_{\text {sum }}=\psi_{1}+\psi_{2}$ of these waves is also a solution of the Schrödinger equation, and calculating the squared amplitude of the superposition using $e^{i \vartheta}=\cos \theta+i \sin \theta$, $e^{-i \vartheta}=\cos \theta-i \sin \theta$, gives

$$
\begin{aligned}
R^{2}=\psi_{\text {sum }} \psi_{\text {sum }}^{*} & =\left(R_{1} e^{\frac{i S_{1}}{\hbar}}+R_{2} e^{\frac{i S_{2}}{\hbar}}\right)\left(R_{1} e^{-\frac{i S_{1}}{\hbar}}+R_{2} e^{-\frac{i S_{2}}{\hbar}}\right) \\
& =R_{1}^{2}+R_{2}^{2}+2 R_{1} R_{2} \cos \left[\frac{2 \pi\left(S_{1}-S_{2}\right)}{h}\right]
\end{aligned}
$$

The third term on the right describes the interference between the superposed waves ${ }^{3}$, and its sign is variable depending on the phase difference [2]. Using the standard measure of $h$ and expressing $\cos \left[\frac{2 \pi\left(S_{1}-S_{2}\right)}{h}\right]$ in the form $\cos (2 \pi \mathrm{n}+\theta)$, where $\mathrm{n}$ is an integer and $0 \leq \theta \leq 2 \pi$, then $\cos \left[\frac{2 \pi\left(S_{1}-S_{2}\right)}{h}\right]=\cos (2 \pi \mathrm{n}+\theta)=\cos \theta^{4}$ and equation (2a) simplifies to

$$
R^{2}=R_{1}^{2}+R_{2}^{2}+2 R_{1} R_{2} \cos \theta
$$

The remarkable feature of Equation (2) is that, as is well known, the cosine is mathematically equivalent to and has all the attributes of a coefficient of correlation [3]. Thus, from the standard result in statistics for the variance of the sum of two correlated random variables [4], the squared amplitude is mathematically equivalent to this variance, where the two variables have variance $R_{1}^{2}$ and $R_{2}^{2}$ and a correlation coefficient $\cos \theta$.

\footnotetext{
${ }^{1}$ The integration is over the support of $\psi \psi^{*}$ if different from $-\infty \leq x \leq \infty$.

${ }^{2}$ Time $t$ is process or experiment specific, such as time since a particle was emitted or since an experiment commenced.

${ }^{3}$ Note the shift from $\hbar$ to $h / 2 \pi$.

${ }^{4}$ Using $\cos (\alpha+\beta)=\cos \alpha \cos \beta-\sin \alpha \sin \beta$.
} 
There are also interference terms in the momentum field determined by $\psi_{\text {sum }}$ [2], but we are interested in the position field and if we specify $x$, we cannot specify a complementary momentum $p$ without violating the uncertainty relationship $\sigma_{x} \sigma_{p} \geq \hbar / 2$, and a focus on the position of a particle means that there is no need, nor any way, to also consider momentum.

\section{$2 \quad$ What might equivalence between $\psi_{\text {sum }}(x, t) \psi_{\text {sum }}^{*}(x, t)$ and a variance signify?}

John von Neumann believed [5] that quantum randomness cannot be reduced to a statistical variation of properties in an ensemble of systems in the way classic randomness is, and likewise Richard Feynman [6] believed that probability interference in quantum mechanics showed that the latter describes statistical properties in microscopic phenomena, where classic Kolmogorov probability theory is not applicable. Later, no-go theorems such as Bell's theorem confirmed that one cannot reproduce quantum probabilities from classic probability theory, but the possibility nevertheless remains that hidden variables may exist that would bypass this limitation.

In work from 1996 to 2012, Vladimir Man'ko and others [7] were able, working only with classic Kolmogorov probabilities, to reproduce quantum probability results. They showed how to describe quantum states using a positive probability distribution called a symplectic tomogram, which is a nonnegative function $W(x, \mu, v)$ of the random position $x$ measured in reference frames in phase space with rotated and scaled axes $q \rightarrow \mu q, p \rightarrow v p$ where $\mu=e^{\lambda}$ $\cos \theta, v=e^{-\lambda} \sin \theta, \theta$ is the angle of rotation and $e^{\lambda}$ is the scaling parameter.

Quantum mechanics is a physical system that is described in probabilistic terms, and despite this achievement by Man'ko and his colleagues, it is nevertheless impossible to ignore the paradox presented by the coincidence that mathematically, the squared amplitude of superposed wavefunctions has the form of a variance of the sum of correlated random variables, not the form of a probability.

The undoubted empirical success of quantum mechanics establishes that after normalization, the squared amplitude of two superposed wavefunctions gives the probability density $f(x, t)$ associated with the particle whose motion is described by the superposition being at the point $(x, t)^{5}$, but the paradox suggests that there may be a reconciliation between quantum and classic probability that is different from the tomographic model and that there may be deeper physics within quantum mechanics. This article will explore how the paradox might be resolved.

First, it is useful to picture a quantum mechanical process such as the self-interference of electrons sent one at a time through a two-slit interferometer to a detection screen. First, after 100 electrons have been dispatched, there will be no discernible pattern, but after 100,000 electrons have been dispatched, a pattern of multiple bands of dense detection separated by sparse detection will take shape across the entire detection screen. These bands of constructive and destructive interference and the varying detection intensity within the bands

\footnotetext{
${ }^{5}$ For economy of notation, $(x, t)$ denotes a position between $x$ and $x+d x$ at time $t$.
} 
from peak to trough will become increasingly distinct as the cumulative number of electrons sent through the interferometer increases.

Likewise, before normalization, the squared amplitude relating to a quantum mechanical process as described by its wavefunction represents the mean number of detections of a phenomenon in the interval between $x$ and $x+d x$ at experiment time $t$ resulting from many experiments. In the case of the self-interference example, the squared amplitude is proportional to the build-up of particle detection intensity at $(x, t)$, and when normalized by dividing it by the sum of the squared amplitudes over all $x$ at time $t$ is the expected relative frequency of detections there, and the probability of finding a particle at $(x, t)$ from one trial.

It is instructive to recall a little of what von Weizsäcker covered in his 1973 paper on probability and quantum mechanics [8]. Noting that a single measurement will not suffice to convince us whether a result is to be considered a confirmation or a refutation of what we expected and we will repeat the measurement many times and apply the theory of errors, he summarized his analysis of the structure of an empirical test of a theoretical prediction in an abbreviated statement by saying "An empirical confirmation or refutation of any theoretical prediction is never possible with certainty but only with a higher or lesser degree or probability." He pointed out that in practice, every application of the theory of errors implies that we consider relative frequencies of events to be predictable quantities. He said that in this sense, probability is a measured quantity and that his abbreviated statement also applies to probability itself, leading him to the conclusion that the empirical test of a theoretical probability is only possible with some degree of probability. At the conclusion of his paper, von Weizsäcker expressed the belief that "there is a quantum theory behind quantum theory, precisely because probabilities can only be defined with the help of probabilities".

Taking these observations into account, a starting point toward explaining why the squared amplitude of two superposed waves has the form of a variance of the sum of two correlated random variables and not the form of a probability would therefore be to suppose that before normalization, the squared amplitude is associated with a hidden variable whose mean equals the variance of another closely related and relevant hidden variable, a property we call the mean/variance property. Finding a pair of such variables with the properties needed to be consistent with quantum mechanics is the purpose of this article.

\section{Properties that must be satisfied and the formulation of the variables}

\section{The necessary properties}

The search begins with a statement of the properties that such a pair of hidden variables must display to comply with quantum mechanics. We call the generic form of these variables the unit base variable and the unit squared amplitude variable, respectively. Their necessary properties are that:

1. The unit base variable must have a mean of zero and variance of one, and the unit squared amplitude variable must be the square of the unit base variable in order that its mean equals 1 , the variance of the unit base variable (the mean/variance property). 
2. The element of a wavefunction which is directly relevant to equation (2b) and capable of having these properties is its real part, the product of its amplitude and the cosine of its argument, which captures its essence. Accordingly, the unit base variable must be the product of two independent variables, an amplitude variable, and a cosine variable, and providing that the properties in 1 above are satisfied, the unit squared amplitude variable forms a pair with the closely related unit base variable.

3. These two generic variables must be transformed by scaling to quantum processspecific variables wherever there is quantum activity:

a. The transformed unit squared amplitude variable, which we call the squared amplitude variable, is the stochastic analogue of the deterministic squared amplitude, and its transforming scale factor and hence its mean equals the deterministic squared amplitude.

b. The transforming scale factor of the unit base variable is the square root of the scale factor of the squared amplitude variable, making the transformed base variable the stochastic analogue of the square root of the deterministic squared amplitude.

4. The mean of the squared amplitude variable not only equals the deterministic squared amplitude but must also equal the variance of the transformed base variable.

5. The squared amplitude variable must accommodate nonlocal and entangled quantum activity as well as local activity ${ }^{6}$.

As a result of these properties, the Law of Large Numbers ${ }^{7}$ will ensure that with repeated experiments under the same conditions, the mean of the realizations of the squared amplitude variable converges to the deterministic squared amplitude as formulated in the causal interpretation of quantum mechanics.

\section{The formulation}

The search for variables that have the properties that the task requires immediately leads to the following set, with the probability distributions of the dependent variables $W$ and $Z$ in 2 and 3 below being given in Sections 4 and 5 respectively:

1. A generic variable $Z$ that is the square of another generic variable $W$, which is the product of two independent seed variables $A$ and $C$ :

a. Variable $A \sim U(0,3)$, which is a generic stochastic analogue of the amplitude of a wavefunction, and

b. Variable $C \sim U(-1,1)$, which is a generic stochastic analogue of the cosine of the argument of a wavefunction ${ }^{8}$.

\footnotetext{
${ }^{6}$ Which means that the scaled unit base variable will do likewise.

${ }^{7}$ The law states that the average of the results obtained from a large number of trials should be close to the expected value and will tend to become closer to the expected value as more trials are performed.

${ }^{8}$ The function $\theta=\cos ^{-1} C$ where $C \sim U(-1,1)$ gives the pdf of the associated random argument $\theta$ i.e., $f_{\vartheta}(\theta)=\frac{1}{4}|\sin (\theta)|,(0<\theta<2 \pi)$.
} 
2. The dependent unit base variable $W$ is the zero mean/unit variance generic stochastic analogue of the real part of a wavefunction, properties that come from the supports of $A$ and $C$.

3. The dependent unit squared amplitude variable $Z$ is the unit mean generic stochastic analogue of the square of the real part of a wavefunction.

4. When and where a quantum process is active, the unit generic variables $W$ and $Z$ instantaneously transform through scaling at every applicable point $(x, t)$ into specific stochastic processes. The transforming scale factor for $\mathrm{Z}$ is $S F$ and for $W$ is $\sqrt{S F}$, where $S F$ equals the deterministic squared amplitude of the process. As a result:

a. $W$ transforms into the process-specific stochastic analogue of the square root of the squared amplitude of the wavefunction, denoted $\mathrm{X}^{9}$.

b. $Z$ transforms into the squared amplitude variable, the process-specific stochastic analogue of the squared amplitude of the wavefunction, denoted $\mathrm{Y}$.

\section{Remark}

The generic variables that culminate in the unit squared amplitude variable $Z$ are not linked to any point in space or time. It seems that $Z$ describes a regular simultaneous vibration-like phenomenon throughout the the universe, effectively a universal clock in which the intensity of the phenomenon varies randomly from instant to instant with points on the support of $Z$, $0<z \leq 9^{10}$ being in correspondence with its intensity, and with each realization $z$ (s) at universal time $s$ on the support present everywhere in the universe in the same instant ${ }^{11}$. In the instant $d s$ on the clocks of the set of active quantum processes synchronized with universal time $s$, the same realization $z$ (s) would be instantly transformed by scale factors equal to the deterministic squared amplitudes of those processes, restated from time $t$ to time $s^{12}$, into the universal set of active squared amplitude variables and their stochastic processes.

\section{The base variable}

\section{The unit base variable}

The distribution of the product of two independent uniform variables, here $A$ and $C$, whose product forms the variable $W$, is known [9], and in this case, pdf is (see Appendix A):

$$
f_{W}(w)=-\left(\frac{1}{2}\right)\left(\frac{1}{3}\right) \ln \left(\frac{|w|}{3}\right),(-3 \leq w \leq 3)
$$

$W$ is i.i.d. at all points $x$ at time $t$. The mean and variance of pdf (3a) are 0 and 1, respectively, as required (see Appendix A). The cumulative density function is also needed for simulation and goodness of fit testing (see the next subsection).

By symmetry of pdf (3a), the cumulative density, in a form that is useful in stepping around the singularity at $w=0$, is as follows (see Appendix A):

\footnotetext{
${ }^{9}$ The sign of $\mathrm{X}$ follows the sign of $W$.

${ }^{10} W$ is the product of $A$ and $C$ so has a support of $-3 \leq w \leq 3$ and $Z$, the square of $W$. has a support of $0<z \leq 9$.

${ }^{11}$ Possibly unaffected by the influences of general relativity near massive bodies.

${ }^{12} \mathrm{See}$ the further discussion on this point in Section 5.
} 


$$
F_{W}(|w|)=-\left(\ln \left(\frac{|w|}{3}\right)-1\right) \frac{|w|}{3} \cdot(0<|w| \leq 3)
$$

The scaled base variable

The transformation to the specific variable $\mathrm{X}$ when and where a quantum process is active is achieved by multiplying the unit base variable by the scale factor $\sqrt{S F}$, where

$$
S F=R_{1}^{2}(x, t)+R_{2}^{2}(x, t)+2 R_{1}(x, t) R_{2}(x, t) \cos \theta(x, t)
$$

which is the scale factor that is applied to the squared amplitude variable (see Section 5). From (3a) and using the standard method for deriving the probability density of a function of a random variable, the scaled base variable pdf is:

$$
f_{W(\sqrt{S F})}(\sqrt{S F} w)=-\left(\frac{1}{6 \sqrt{S F}}\right) \ln \left(\frac{|w|}{3 \sqrt{S F}}\right),(-3 \sqrt{S F} \leq w \leq 3 \sqrt{S F})
$$

The transformed pdf (4a) has a mean of 0 and variance of $S F$, which equals the mean of the squared amplitude variable as required (see Section 5). Scaling the unit base variable cdf (3b) is needed for simulation testing to check for correctness within a margin that allows for randomness in the simulation. This is done in a manner ready for squaring when the squared amplitude variable is tested and involves multiplying the absolute value of the simulated $W$ data by the scale factor $\sqrt{S F}$ to put it in the form $\sqrt{S F}|\mathrm{w}|$, transforming the unit cdf (3b) to the scaled base variable cdf (4b) below, and testing it as described in Appendix C:

$$
F_{W(\sqrt{S F})}(\sqrt{S F}|w|)=-\left(\ln \left(\frac{|w|}{3 \sqrt{S F}}\right)-1\right) \frac{|w|}{3 \sqrt{S F}},(0<|w| \leq 3 \sqrt{S F})
$$

\section{The squared amplitude variable}

\section{The unit squared amplitude variable}

In this formulation $W$ and $Z$ are always present everywhere. $Z$ is the square of $W$, and the distribution of the square of a random variable is well known and in this case is pdf (5a) below (see Appendix B):

$$
f_{Z}(z)=-\frac{1}{6 \sqrt{z}} \ln \frac{\sqrt{z}}{3},(0<z \leq 9)
$$

The variable $Z$ is i.i.d. at all points $x$ at time $t$. The mean of $Z$ is 1 , which agrees with the variance of the unit base variable, as required. Its variance is $56 / 25=2.24$ (see Appendix B), and its coefficient of variation is $\sqrt{2.24} \cong 1.5$, or $150 \%$, compared with, for example, $100 \%$ for a gamma distribution with a shape parameter of 1 . As with the base variable, the cumulative density function is needed for simulation and goodness of fit testing and because like pdf (5a) it is important. The cumulative density of (5a) is cdf (5b) (see Appendix B):

$$
F_{Z}(z)=-\left(\ln \left(\frac{\sqrt{z}}{3}\right)-1\right)\left(\frac{\sqrt{z}}{3}\right),(0<z \leq 9)
$$

\section{The squared amplitude variable}

As with the base variable, the transformation to the squared amplitude variable $\mathrm{Y}$ when and where a quantum process is active is achieved by multiplying the unit variable by the scale 
factor $S F$ defined in the previous section and below, which aligns the mean of the squared amplitude variable with the deterministic squared amplitude. The scaled pdf is:

$$
f_{Z(S F)}(S F Z)=-\frac{1}{6 \sqrt{Z S F}} \ln \left(\frac{1}{3} \sqrt{\frac{z}{S F}}\right),(0<z \leq 9 S F)
$$

The scale factor $S F=R(x, t)^{2}=R_{1}^{2}(x, t)+R_{2}^{2}(x, t)+2 R_{1}(x, t) R_{2}(x, t) \cos \theta(x, t)$, which provides the link to the point $(x, t)$. If there is quantum activity but no interference, $S F$ equals one or another of $R_{1}^{2}(x, t)$ or $R_{2}^{2}(x, t)$. Note that time $t$ is process or experiment specific, such as time since a particle was emitted.

The generic unit squared amplitude variable $Z$ is present in the background everywhere all the time and is transformed to the squared amplitude variable $Y$ across the set of stochastic processes behind all the active quantum events at any time. Thus, $Z$ is the generic archetype we set out to find.

As remarked on in Section 3, if the clocks of $N$ active processes $Y(j), j \in[1, N]$ were synchronized with a universal clock and the realization of $Z$ in accord with pdf (5a) at time $S$ is $Z(\mathrm{~s})$, then the realization of $\mathrm{Y}(j, x, s)$ for active process $j$ at that time is $R(j, x, s)^{2} z(s)$. The $N$ active processes $j=1, \ldots \ldots, N$ are simultaneously active at universal time $s$, but though we know that the distribution of $z$ (s) follows pdf (5a) regardless of the specific time $s$ and has a mean of 1 and a variance of 2.24, we know nothing about $s$ in an absolute sense, hence nothing about $R(j, x, s)^{2}$, but we can assume that each $d s$ is synchronous with each $d t$ and we can in practice work with process-specific time $t$ and the realization of $\mathrm{Y}(j, x, t)$ in it.

Where quantum process $j$ becomes active, the unit base variable $W$ is instantly transformed by the scale factor $\sqrt{\sum S F(j, x, t)}$ to the variable $\mathrm{X}(j, x, t)$ and the unit squared amplitude variable $Z$ by the scale factor $S F(j, x, t)$ to the squared amplitude variable $\mathrm{Y}(j, x, t)$. Using shortened notation ${ }^{13}$, the mean of $Y(j)$ equals the scale factor $S F(j)$, which equals the formulation of the squared amplitude of process $j$ in quantum mechanics. The mean of $Y(j)$ also equals the variance of $\mathrm{X}(j)$, satisfying the mean/variance property.

In summary, $Z$ is an enduring and universal process, and the transformed $\operatorname{set} Y(j), j \in[1, N]$ comprises the universe of active processes, with each member specifying the details of its process at every applicable point $x$ at the process time $t$ on its clock. In particular, the probability that a particular particle in a particular process $j$ is at a particular point $x$ at a particular time $t$ on its clock is the normalized variable $\mathrm{Y}(j)$ and has a mean equal to the normalized squared amplitude as formulated in quantum mechanics.

In addition to pdf (6a), the squared amplitude cdf is also needed for simulation testing to check for correctness. This involves squaring the simulated $W$ data, transforming the unit cdf (5b) to the scaled cdf (6b) below and testing it as described in Appendix C:

$$
F_{Z(S F)}(S F Z)=-\left(\ln \left(\frac{1}{3} \sqrt{\frac{z}{S F}}\right)-1\right)\left(\frac{1}{3} \sqrt{\frac{z}{S F}}\right),(0<z \leq 9 S F)
$$

\footnotetext{
${ }^{13}$ Where the context permits $S F(j, x, t), \mathrm{X}(j, x, t)$ and $\mathrm{Y}(j, x, t)$ will be denoted $S F(j), \mathrm{X}(j)$ and $\mathrm{Y}(j)$.
} 
Note that the scaled cdf reaches a value of 1 when $z=9 S F$, where $S F$ can be any positive number, making pdf (6a) and cdf (6b) capable of support anywhere in the range $0<z<\infty$. This means that the squared amplitude variable can be compressed with $0<S F<1$ or expanded with $S F>1$ to match the deterministic squared amplitude, whatever its size. This is illustrated in Table 1, where the cumulative density in the first bin of a set of bins of equal width is shown for a range of scale factors ${ }^{14}$.

\begin{tabular}{|c|l|l|l|l|l|l|l|l|l|l|l|}
\hline \multicolumn{10}{|c|}{$\begin{array}{c}\text { Pable 1 } \\
\text { Percentage of the cumulative density of the squared amplitude variable Y } \\
\text { in the first bin of a set of bins of equal width } 0.25\end{array}$} \\
\hline$S F$ & 72 & 36 & 18 & 9 & 3 & 1 & .72 & .3 & .18 & .09 & .03 \\
\hline \begin{tabular}{c} 
(rounded) \\
\hline
\end{tabular} & 9.7 & 12.7 & 16.6 & 21.6 & 32.1 & 46.5 & 51.6 & 66.6 & 76.0 & 88.2 & 99.9 \\
\hline
\end{tabular}

\section{The significance of the squared amplitude variable}

The identification of a squared amplitude variable that satisfies the properties needed to be compatible with the formulation of quantum mechanics suggests the possibility that the formulation of probability in quantum mechanics could be pointing to and employing the normalized mean of a random variable, with the average of the realized squared amplitude variable from many experiments converging to that mean. Quantum probability would then be a prediction of the expected relative frequencies of possible experimental outcomes, which is the very definition of a probability. What is suggested here is that a squared amplitude variable and its stochastic process originate from a generic stochastic variable $Z$, which is an archetype in the Platonic concept of pure form, satisfying the properties in Section 3 and embodying the essential characteristics of a precursor variable of quantum probability. Under this formulation, quantum mechanics provides a prediction of the relative frequency of a quantum event occurring at a spatial point and experiment time, but to paraphrase von Weizsäcker the basis of this prediction can only be defined with the help of relative frequencies i.e., probabilities.

Along such lines, the average over repeated experiments of the relative frequency of realizations of the squared amplitude variable at a spatial point $x$ at experiment time $t$ i.e., the number of realizations there and then as a fraction of the total of all such realizations over all possible spatial points at that time, would if known be seen to converge to the probability predicted by quantum mechanics that the particle whose motion is governed by the wavefunction is at $x$ at time $t$, and the average, over repeated experiments, of the mean particle position calculated using the realized relative frequency at each possible spatial point at that time, would if known be seen to converge to the expected value of the particle position that is predicted by quantum mechanics. Of course, these realizations are not countable or known, but can be hypothesized to exist.

\footnotetext{
${ }^{14}$ Note that in simulation testing the number of bins of width $b w$ used should be $9 S F / b w$.
} 


\section{Simulation and goodness of fit testing of the squared amplitude variable}

To test the fit between expected and simulated results, the squared amplitude variable was simulated using 5000 simultaneous random realizations of the amplitude-like and cosine seed variables $A$ and $C$, with realizations of the scaled base and squared amplitude variables $\mathrm{X}$ and $\mathrm{Y}$ calculated from them: in the case of $\mathrm{X}$ by multiplying the $A$ and $C$ simulated data and then multiplying the absolute values of the result by $\sqrt{S F}$ to get simulated $\sqrt{S F}|W|$ data; and in the case of Y, by squaring the simulated $\sqrt{S F}|W|$ data to get simulated $S F W^{2} \equiv S F Z$ data, then testing the results against those expected as described in Appendix C.

The simulated values provide an independent test of the derivation of the squared amplitude variable $Y$. The three charts that follow compare the simulated results with those expected using the cumulative density function (6b), and Table 2 following the charts provides some key statistics.

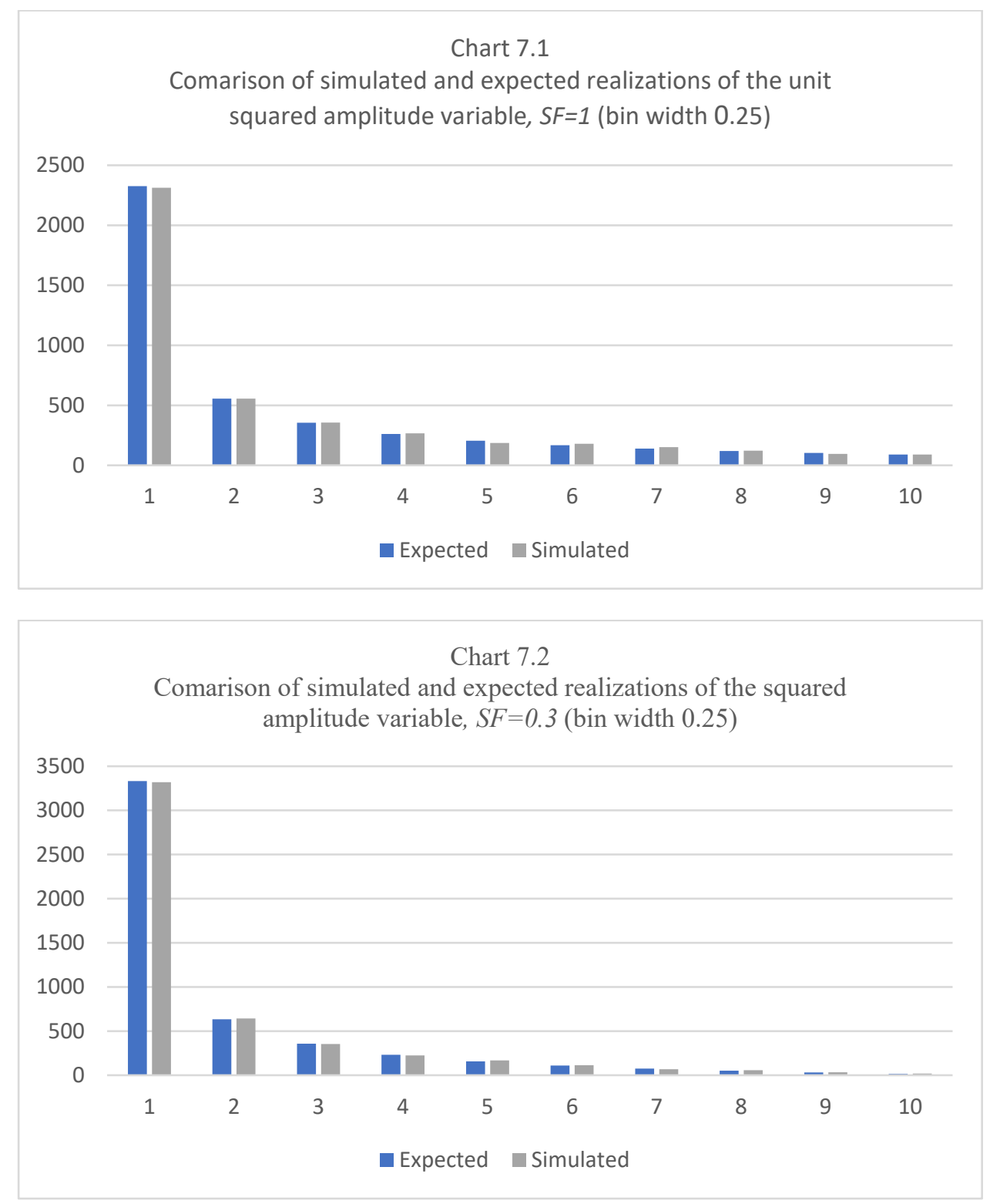


Chart 7.3

Comarison of simulated and expected realizations of the squared amplitude variable, $S F=3$ (bin width 0.25 )

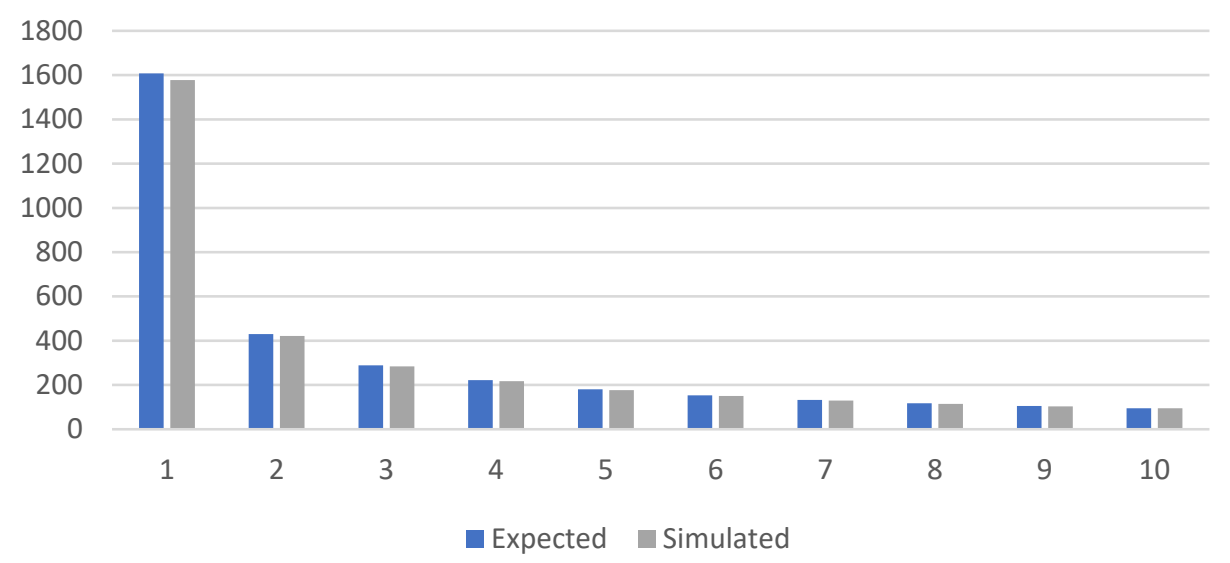

\begin{tabular}{|c|c|c|c|c|c|c|}
\hline \multicolumn{7}{|c|}{$\begin{array}{c}\text { Table } 2 \\
\text { Key statistics }\end{array}$} \\
\hline Variable & $\mathrm{X}$ & $\mathrm{Y}$ & $W$ & $Z$ & $X$ & Y \\
\hline $\begin{array}{l}\text { Scale factor }(S F) \\
\text { (Y is } Z \text { scaled by } S F, \mathrm{X} \text { is } W \text { scaled by } \sqrt{S F} \text { ) }\end{array}$ & \multicolumn{2}{|c|}{3} & \multicolumn{2}{|c|}{$\begin{array}{c}\text { Unscaled } \\
\text { unit variables }\end{array}$} & \multicolumn{2}{|c|}{.3} \\
\hline Expected mean & 0 & 3 & 0 & 1 & 0 & .3 \\
\hline Simulated mean & .002 & 3.04 & .001 & 1.01 & .001 & .304 \\
\hline Expected variance & 3 & 20.16 & 1 & 2.24 & .3 & .2016 \\
\hline Simulated variance & 3.04 & 20.48 & 1.01 & 2.28 & .304 & .2048 \\
\hline
\end{tabular}

It can be seen from the charts that there is a good fit of the results expected from the derived squared amplitude variable with the results of the simulations. Appendix $\mathrm{C}$ provides the data underlying the charts and describes the method used. The charts show comparisons for the first ten bins of width 0.25 , starting with the 0 to 0.25 bin on the left.

It can also be seen from the key statistics in Table 2 that the scaled base and squared amplitude variables have the required properties set out in Section 3, including the essential mean/variance property. The table confirms a good fit between expected and simulated means and variances, including the expected and simulated variances of the squared amplitude variable, which have been included as a matter of interest.

\section{Consistency of the formulation with nonlocality and entanglement}

In our formulation the generic variable $\mathrm{Z}$ is present everywhere all the time. As discussed above, it is transformed by local quantum activity into a specific variable $\mathrm{Y}$ in a localized stochastic process. However, $\mathrm{Z}$ is a universal hidden variable which potentially makes it capable of bypassing Bell's theorem. This means that Y could also apply to interactions such as measurements relating to two entangled particles that are too far apart in space and too close together in time for them to be connected even by signals moving at the speed of light. 
This is possible because such interactions can nevertheless be physically connected through Holland's quantum theory of motion, as described in his account of the de Broglie-Bohm causal interpretation of quantum mechanics and his conception of a many-body system [10].

Holland defines an individual $n$-body system as follows:

a) A wavefunction $\psi=\psi\left(\mathbf{x}_{1}, \ldots, \mathbf{x}_{n}, t\right)$ is defined in a $3 n$-dimensional configuration space in which $\mathbf{x}_{1}, \ldots \ldots, \mathbf{x}_{\mathbf{n}}$ provides a set of rectangular Cartesian coordinates.

b) A set of $n$ point particles pursuing trajectories $\mathrm{x}_{i}(t), i=1, \ldots, n$ in threedimensional Euclidean space. A single configuration space trajectory is equivalent to $n$ particle trajectories in Euclidean space.

As Holland explains, a many-body system then means a single wavefunction with a set of particles, with no individual wave associated with a particle. While the particles each move in 3 -space, the guiding wave is defined in $3 n$-space. Because the wave in this interpretation has a physical influence on the particles, the configuration space is attributed as much physical reality as that of Euclidean space in the one-body theory. These features in Holland's extension of his quantum theory of motion to many-body systems, namely that an individual physical system resides in a multidimensional configuration space and that the latter is physically real, are in Hollands words "striking features not evident in the one-body case" [10].

Holland points out that this conception, with these features, including that the configuration space wavefunction depends on a single evolution parameter $t$, implies that the state of the $n$ particles is specified at a common time and that there is a nonlocal connection between them, with the instantaneous motion of any one particle depending on the coordinates of all the other particles at the same time. If part of the system is disturbed in a localized region of three-dimensional space, the configuration space will respond, and consequently, all the particles making up the system will be affected instantaneously [10]. Holland studies the conditions in a two-body system under which we can expect to find correlations in particle motions and the conditions under which they are independent [11]. The case where $\psi\left(\mathbf{x}_{1}, \mathbf{x}_{2}, t\right)$ is factorizable and is the product of two wavefunctions associated with each of the particles is one in which the two particles are physically independent. To examine a case with correlation and entanglement, Holland moves to a wavefunction expressed as the sum of two such factorizable wavefunctions [11]:

$$
\psi\left(\mathbf{x}_{1}, \mathbf{x}_{2}, t\right)=\psi_{A}\left(\mathbf{x}_{1}, t\right) \psi_{B}\left(\mathbf{x}_{2}, t\right)+\psi_{C}\left(\mathbf{x}_{1}, t\right) \psi_{D}\left(\mathbf{x}_{2}, t\right)
$$

Noting that such solutions may be constructed when there is no classic interaction between the particles, Holland states that when the summands in (8a) overlap in configuration space, that is, when $\psi_{A} \cap \psi_{C} \neq \varnothing$ and $\psi_{B} \cap \psi_{D} \neq \varnothing$, the wavefunction is nonfactorizable and entangled [11], and he gives the joint squared amplitude of (8a) in Euclidean space to be as follows [11] ${ }^{15}$ :

$$
R^{2}=R_{A}^{2} R_{B}^{2}+R_{C}^{2} R_{D}^{2}+2 R_{A} R_{B} R_{C} R_{D} \cos \left[\left(S_{A}+S_{B}-S_{C}-S_{D}\right) / \hbar\right]
$$

${ }^{15}$ Which when normalized is the joint probability. 
and states that where the interference term in $(8 b)$ is finite the particle motions are nonlocally correlated, and a particle is no longer associated with wave $\psi_{A} \psi_{B}$ or wave $\psi_{C} \psi_{D}$, but instead both particles are guided by one wavefunction $\psi\left(\mathbf{x}_{1}, \mathbf{x}_{2}, t\right){ }^{16}$. To quote Holland, "bearing in mind that the primary property of the wavefunction is the influence it exerts on the particles, we deduce that the particles are statistically correlated because they are physically connected". When the cosine term in (8b) is simplified to $\cos \theta$, as was done in Section 1 , and the squared amplitude ( $8 \mathrm{~b})$ is used as the scale factor $S F$ in pdf (6a), Y becomes a specific variable and stochastic process which is the stochastic analogue of the squared amplitude (8b), including its inherent nonlocality. Thus, the universal generic variable $Z$, the process-specific variable $Y$, and their formulation are consistent with nonlocality in the causal interpretation of quantum mechanics as it is embodied in Holland's quantum theory of motion and encompass both local and nonlocal quantum events.

\section{Conclusion}

This article presents a formulation in which behind the squared amplitude of either a superposed or individual wavefunction as formulated in the causal interpretation there is an associated specific variable $Y$ at each applicable point in space and time that originates from a universal generic archetypical variable $Z$. The variable $Y$ has a mean at each point and time that equals, and whose average realization over repeated trials of an experiment converges to, the squared amplitude. When the counts of realizations at each point and time are normalized to their relative frequencies, their averages converge with repeated trials to the set of probabilities predicted by the causal interpretation of quantum mechanics that the particle whose motion is described by its wavefunction is at these points, and the average of the mean particle position calculated using these relative frequencies likewise converges to the expected value predicted by quantum mechanics. Importantly, as shown in the previous section, despite being developed using classic probability theory, the variable $\mathrm{Y}$ and its stochastic process can relate to either a local or a nonlocal quantum mechanical process.

Quantum mechanics works perfectly well without a mathematical reconciliation between quantum probability and the axioms of probability. However, the results provide insight that quantum probability itself could originate from a generic universal stochastic archetype that triggers a specific variable when and where there is quantum activity which continues as a stochastic process while the activity continues. When normalized, this variable would be a stochastic analogue of quantum probability, a prospect that not only raises intriguing questions about the nature of the underlying physics that could be described by such a process, but that also might inform or otherwise prove useful in quantum technology. In particular, the mathematics seem to describe a regular simultaneous vibration-like phenomenon of variable intensity throughout the universe which transforms into processspecific squared amplitude variables and their stochastic process wherever quantum processes are active.

\footnotetext{
${ }^{16}$ In this article, the $R$ and $S$ terms are functions of position at time $t$ of the particles 1 and 2 on their single spatial dimension lines $x_{1}, x_{2}$ and the particles are guided by $\psi\left(x_{1}, x_{2}, t\right)=\psi_{A}\left(x_{1}\right) \psi_{B}\left(x_{2}\right)+\psi_{C}\left(x_{1}\right) \psi_{D}\left(x_{2}\right)$.
} 


\section{APPENDIX A}

The unit base variable

To derive the pdf of the product of the two independent uniform random variables, $A \sim U(0,3)$ and $C \sim U(-1,1)$ begin with the probability density of a $\Gamma(2,1){ }^{17}$ distribution $f(s) d s=$ $s e^{-s} d s(0<s<\infty)$. Let $s=-\ln w$ so that $d s=-d(\ln w)=-d w / w(0<w<1) \rightarrow$ $f(s)=-\ln w(0<w<1)$. As Huber saw [9], this causes larger values of $s$ to lead to smaller values of $w$ so that when the substitution is reversed, a minus sign must be attached to the result. Thus:

$$
f(s) d s=-\left(-\ln w e^{-(-\ln w)}(-d w / w)\right)=-\ln w d w,(0<w<1)
$$

Allowing for the support of $A$ gives $-\ln (w / 3) d(w / 3)=-\frac{1}{3} \ln (w / 3) d w(0<w<3)$. Replacing $w$ with $|w|$ and spreading the pdf across $-3<w<3$ gives ${ }^{18}$ :

$$
f_{W}(w)=-\frac{1}{2} \frac{1}{3} \ln (|w| / 3),(-3<w<3)
$$

Because of the symmetry of (A1), we can use the following alternate form to derive a useful form of the cumulative density function, using only the positive half of the support:

$$
f_{W}(|w|)=-\frac{1}{3} \ln (|w| / 3),(0<|w| \leq 3)
$$

To integrate (A2), substitute $u=\frac{|w|}{3}$, giving $d|w|=3 d u$ so that (A2) becomes $\int-\ln (u) d u=-\int \ln (u) .1 d u$, treat factor 1 as the derivative of $u$ and integrate by parts giving $\int-\ln (u) d u=u-u \ln u$. Reversing the substitution gives:

$$
F_{W}(|w|)=-\left(\ln \left(\frac{|w|}{3}\right)-1\right) \frac{|w|}{3},(0<|w| \leq 3)
$$

Note that $F_{W}(|w|)=1$ when $w=3$, confirming that (A2) is a probability density. By inspection, the mean of $W$ is zero, and drawing on the means and variances of $A,\left(\frac{3}{2}, \frac{9}{12}\right)$ and $C,\left(0, \frac{4}{12}\right)$ and using the standard result for the variance of the product of two independent variables, the variance of $W$ is:

$$
\left(\sigma_{A}^{2}+\mu_{A}^{2}\right)\left(\sigma_{C}^{2}+\mu_{C}^{2}\right)-\mu_{A} \mu_{C}=\left(\frac{9}{12}+\frac{9}{4}\right)\left(\frac{4}{12}\right)=1
$$

\footnotetext{
17 The negative logarithm of a $U(0,1)$ variable has an exponential distribution with rate 1 , so the negative log of the product of two of them has the distribution of the sum of two exponential variables.

As the exponential with rate 1 is a $\Gamma(1,1)$ variable by adding the shape parameters the sum of two of them is $\Gamma(2,1)$.

${ }^{18}$ For a deeper and more explanatory derivation see the elegant treatment by Huber [9].
} 


\section{APPENDIX B}

The unit squared amplitude variable

The density of the square of a random variable is well known and, allowing for symmetry of the variable $W$ that is being squared, is by inspection of pdf (A2):

$$
Z \sim-\frac{1}{6 \sqrt{z}} \ln \frac{\sqrt{z}}{3},(0<z \leq 9)
$$

and

$$
f_{Z}(z)=-\frac{1}{6 \sqrt{z}} \ln \frac{\sqrt{z}}{3},(0<z \leq 9)
$$

To integrate (B2) to provide the cumulative density function, put $u=\frac{\sqrt{z}}{3}$; then $\frac{d u}{d z}=\frac{1}{6 \sqrt{z}}$ giving $\frac{1}{6 \sqrt{z}} d z=d u \rightarrow-\int \ln u d u=u-u \ln u$, and reversing the substitution gives:

$$
F_{Z}(z)=-\left(\ln \left(\frac{\sqrt{z}}{3}\right)-1\right) \frac{\sqrt{z}}{3},(0<z \leq 9)
$$

$F_{Z}(z)=1$ when $z=9$, confirming that (B2) is a probability density.

Using (B2) to integrate for the mean we have:

$-\int \frac{z}{6 \sqrt{z}} \ln \frac{\sqrt{z}}{3} d z=-\int \frac{\sqrt{z}}{6} \ln \frac{\sqrt{z}}{3} d z=-\frac{1}{12} \int \sqrt{z} \ln \frac{z}{9} d z$, put $u=\frac{z}{9} ;$ then $\frac{d u}{d z}=\frac{1}{9}$ and $\frac{1}{9} d z=$ $d u$, also

$\sqrt{z}=3 \sqrt{u}$ and simplying $\rightarrow-27 \int \sqrt{u} \ln u d u$. Integrating by parts, using $\sqrt{u}=\frac{d}{d u} \frac{2 u^{3 / 2}}{3}$ and dropping the factor -27 for the moment gives $\frac{2(\ln u) u^{3 / 2}}{3}-\int \frac{2 \sqrt{u}}{3} d u$, and the latter integration gives $\frac{4 u^{3 / 2}}{9}$. Reversing the substitution and returning the factor -27 gives $-\frac{\left(3 \ln \frac{z}{9}-2\right) z^{3 / 2}}{54}$, and we have:

$$
\mu_{Z}=-\left[\frac{\left(3 \ln \frac{z}{9}-2\right) z^{3 / 2}}{54}\right]_{0}^{9}=1
$$

The same approach is used to integrate for $\mathrm{E}\left[z^{2}\right]$. The substitution $u=\frac{z}{9}$ is the same, and $z^{3 / 2}=27 u^{3 / 2} \rightarrow-243 \int u^{3 / 2} \ln u d u$. Integrating by parts using $u^{3 / 2}=\frac{d}{d u} \frac{2 u^{5 / 2}}{5}$ and dropping the factor -243 temporarily $\rightarrow \frac{2(\ln u) u^{5 / 2}}{5}-\int \frac{2 u^{3 / 2}}{5} d u$, the integration term gives $\frac{4 u^{5 / 2}}{25}$. Reversing the substitution and returning -243 gives $-\frac{\left(5 \ln \frac{z}{9}-2\right) z^{5 / 2}}{150}$ and:

$$
\sigma_{Z}^{2}=\mathrm{E}\left[z^{2}\right]-\mu_{Z}^{2}=-\left[\frac{\left(5 \ln \frac{z}{9}-2\right) z^{5 / 2}}{150}\right]_{0}^{9}-1=\frac{81}{25}-1=\frac{56}{25}=2.24
$$




\section{APPENDIX C}

Data and method for Charts 7.1, 7.2 and 7.3

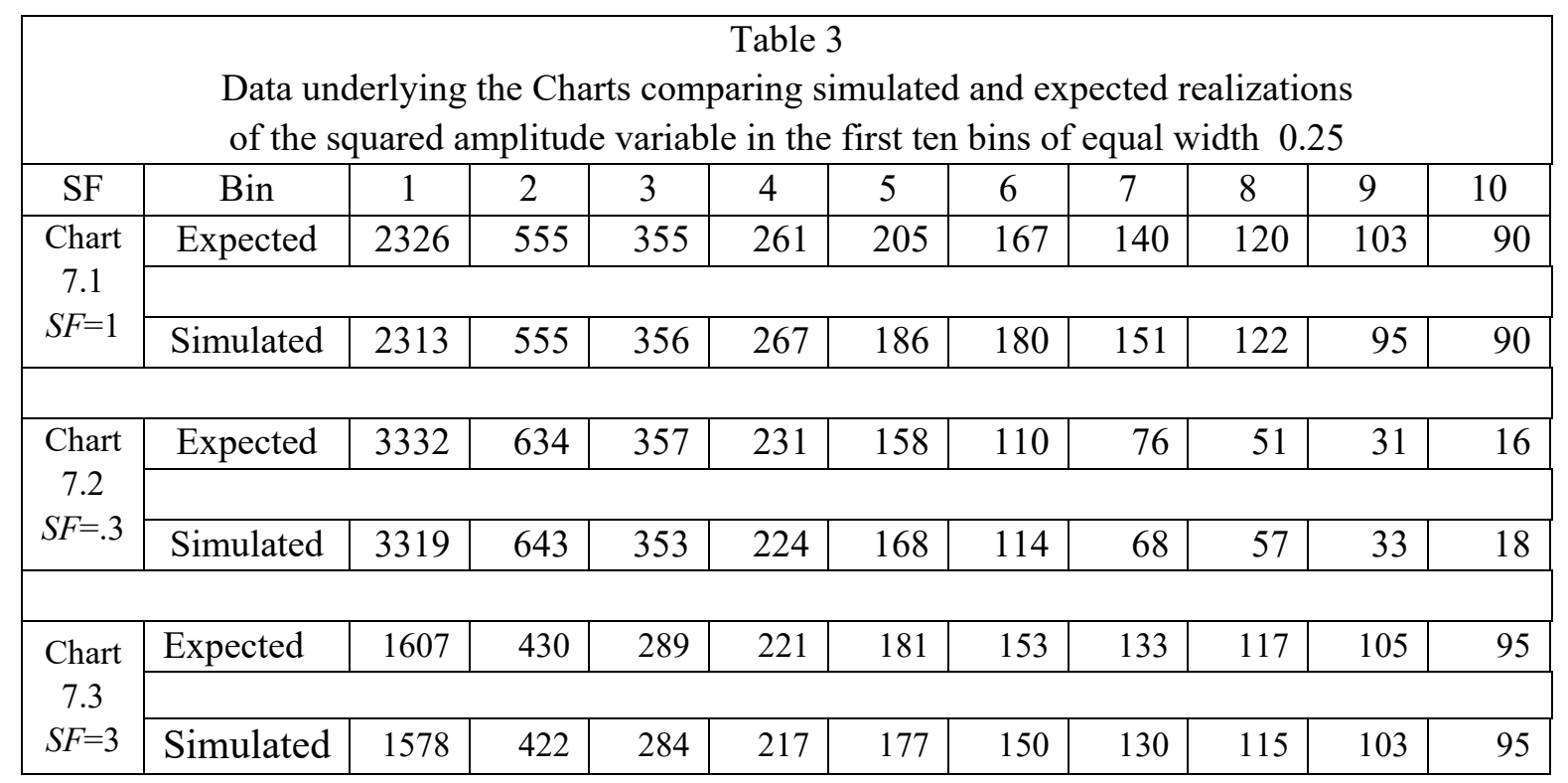

\section{Method of testing the scaled base variable}

The results of simulation testing of the base variable have not been included, but the method used to test cdf (4b) is like that described below for the squared amplitude variable and used to create Table 3 above. As with the squared amplitude variable, testing revealed a good fit. Of course, the cdf and its support, the bin width, and the data transformation $\sqrt{S F}|W|$ referred to in Section 7 are all different, and the application of the method reflects this. For example, the base variable data counted in a particular bin for a particular $S F$ comprise all simulated base variable values $\sqrt{S F}|W|$ that fall in the range of that bin, and the total count in it is compared with the expected count using cdf (4b). Note that because testing the base variable involves simulated data in the form $\sqrt{S F}|W|$ when these data are squared during testing of the squared amplitude variable, they are in the correct form $S F W^{2}$ for that purpose.

\section{Method of testing the squared amplitude variable}

To test cdf $(6 \mathrm{~b})$, there are 5000 simulations and $9 S F / b w$ bins of equal width $b w=.25$ to count the simulated data described in Section 7, progressing from the first bin covering values 0 to $b w$ through the last bin covering values from $(9 S F-b w)$ to $9 S F$, at which point cdf (6b) reaches a value of 1 , as bin width was chosen to give an integer number of bins at this point. Considering the data counted in say the $5^{\text {th }}$ bin for each $S F$, which go from $S F$ to $1.25 S F$, all simulated squared amplitude values $S F W_{5}^{2}$ that fall in the range of this bin for their $S F$ are counted in it, and the total count is compared with the expected count in this bin, which using cdf $(6 \mathrm{~b})$ is $5000\left(F_{Z(S F)}(1.25 S F)-F_{Z(S F)}(S F)\right)$. 


\section{References}

[1] P.R. Holland, The Quantum Theory of Motion: an account of the de Broglie-Bohm causal interpretation of quantum mechanics, vol. Paperback (Cambridge University Press, Cambridge, 1997), pp 67-70

[2] P.R. Holland, The Quantum Theory of Motion: an account of the de Broglie-Bohm causal interpretation of quantum mechanics, vol. Paperback (Cambridge University Press, Cambridge, 1997), pp 86-87

[3] A. Papoulis, Probability, Random Variables, and Stochastic Processes, vol. Paperback (McGraw-Hill Inc., Singapore, 1991), p 154

[4] A. Papoulis, Probability, Random Variables, and Stochastic Processes, vol. Paperback (McGraw-Hill Inc., Singapore, 1991), p 155

[5] J. von Neumann, Mathematische Grundlagen der Quuantenmechanik, (Springer, 1932)

[6] R. Feynman and A. Hibbs, Quantum Mechanics and Path Integrals, (McGraw-Hill, 1965)

[7] V.I. Man'ko et al, On the tomographic picture of quantum mechanics, Physics Letters A: volume 374, issue 26, 7 June 2010, pp 2614-2617

[8] C.F. von Weizsäcker, Probability and Quantum Mechanics, The British Journal for the Philosophy of Science, Vol. 24, No 4, pp. 321-337 (1973)

[9] W. Huber, pdf of a product of two independent Uniform random variables, https://stats.stackexchange.com/q/134896 (version: 2015-01-25), Cross Validated

[10] P.R. Holland, The Quantum Theory of Motion: an account of the de Broglie-Bohm causal interpretation of quantum mechanics, vol. Paperback (Cambridge University Press, Cambridge, 1997), pp 277-282

[11] P.R. Holland, The Quantum Theory of Motion: an account of the de Broglie-Bohm causal interpretation of quantum mechanics, vol. Paperback (Cambridge University Press, Cambridge, 1997), pp 287-290 